\title{
I NUOVI TESTI DI GALENO: TRA EPISTEMOLOGIA E STORIA DELLA CULTURA
}

\author{
Nota del m.e. MARIO VEGETTI (*)
}

(Adunanza del 25 ottobre 2012)

SUNTO. - Il recente ritrovamento di un manoscritto conservato in un convento di Salonicco ha consentito di ampliare la nostra conoscenza dell'opera di Galeno. Nel caso di due opere già note (L'ordine dei miei libri e I miei libri) il nuovo manoscritto permette di colmare una grande lacuna presente nel solo manoscritto finora noto. Il testamento dottrinale di Galeno (Le mie opinioni), finora noto solo nella versione latina di una traduzione araba, è ora leggibile nell'originale greco, e presenta un grande interesse epistemologico. Il nuovo manoscritto ha poi rivelato un'opera del tutto ignota (Sull'imperturbabilità) che costituisce un prezioso documento storico e culturale circa i danni prodotti tanto nelle proprietà di Galeno quanto nelle librerie di Roma dall'incendio del 192.

$$
* * *
$$

ABSTRACT. - The recent discovery of a manuscript in a convent of Thessaloniki has allowed us to expand our knowledge of the work of Galen. In the case of two works already known (The order of my books and On my books) the new manuscript can fill a wide gap in the only mauscript so far available. The doctrinal testament of Galen (On my opinions), so far known only in the Latin version of an Arabic translation, is now readable in the original greek, and has shown a valuable epistemological interest. The new manuscript has also revealed a galenic work completely unkown (How to avoid grief) which is a relevant cultural and historical document about the damages suffered both by the properties of Galen himself and by the Roman libraries in the fire of 192 .

(*) Università degli Studi di Pavia, Italy.

E-mail: mariovegetti@tin.it 
1.

Comincerò dando qualche numero. Il corpus delle opere di Galeno occupa circa 20000 pagine dell'edizione ottocentesca di Kühn, l'unica tuttora completa: si tratta di circa $1 / 8$ di tutta la letteratura in lingua greca che ci è pervenuta da Omero fino all'epoca dello stesso Galeno, cioè alla fine del II secolo dopo Cristo. A questo corpus si aggiungono varie altre opere che ci sono tramandate soltanto nella traduzione araba, o più raramente ebraica e latina. Paul Moraux ha calcolato che nel corso di un cinquantennio di attività letteraria Galeno scrisse circa 500 pagine all'anno: un dato tanto più sbalorditivo se si pensa che oltre al lavoro della scrittura Galeno aveva naturalmente svolto la sua prevalente professione di medico.

Vista l'esistenza di questo sterminato continente di scrittura scientifica e filosofica, del resto ancora in parte inesplorato - nel senso che mancano edizioni critiche, commenti e traduzioni di moltissime opere è legittimo chiedersi se la scoperta di nuovi scritti galenici, totalmente o parzialmente inediti, abbia qualche reale motivo di interesse: non si tratta in fondo che di isolette periferiche rispetto a quel continente. Cercherò di mostrarvi che in effetti qualche motivo c'è. Ma vediamo prima di che cosa si tratta.

2.

Nel 2005 lo studioso francese Antoine Pietrobelli ha ritrovato, in un monastero di Tessalonica, un manoscritto del XV secolo, il Vlatadon 14 (già catalogato ma mai letto nella sua interezza), scoprendo al suo interno quattro operette di Galeno nella loro versione integrale (e affidabile, sulla base del riscontro con passi già noti delle stesse opere).

Si tratta in primo luogo del de ordine librorum suorum (L'ordine dei miei libri) e del de libris suis (I miei libri): due scritti autobibliografici, che costituiscono un unicum in tutta la letteratura filosofico-scientifica antica. Essi erano finora noti grazie a un solo manoscritto, l'Ambrosianus gr. 659, del quale però era caduta una parte, provocando un'ampia lacuna in entrambi gli scritti, che ora è stato possibile colmare. Nella parte ritrovata, c'è un passo interessante per la storia della medicina antica, quello in cui Galeno espone la sua epitome di un trattato del grande anatomista Lykos che finora ci era del tutto ignoto.

Il terzo testo reca la versione greca del de propriis placitis (Le mie opi- 
nioni), una sorta di testamento dottrinale del vecchio Galeno, che finora era noto solo nella versione latina di una traduzione araba. Sebbene magistralmente edito da Vivian Nutton nel Corpus medicorum graecorum, il testo latino lasciava adito a interpretazioni dubbie e fuorvianti.

La quarta operetta è uno scritto epistolare consolatorio, il cui titolo, peri alypias (L'imperturbabilità), ci era noto dal catalogo del de libris suis, ma di cui non restava alcuna traccia: si tratta dunque in questo caso di una vera e propria aggiunta inedita al corpus galenico, che ha, come vedremo, un prezioso valore documentario.

L'edizione critica di de ordine librorum suorum e di de libris suis è stata pubblicata da Véronique Boudon-Millot presso le Belles Lettres nel 2007; quella del peri alypias (de indolentia nel calco latino) da Jacques Jouanna nel 2010. Il de propriis placitis, di cui manca ancora l'edizione critica, è stato pubblicato come editio princeps da V. Boudon e A. Pietrobelli nella «Revue des Etudes grecques» nel 2005. Una edizione migliorata, benché priva di apparato critico, è stata recentemente curata da Alessandro Lami per le edizioni BUR (2012).

\section{Livelli epistemologici delle “opinioni” di GaLeno}

Il vecchio Galeno aveva compiuto un doppio sforzo per consegnare ai contemporanei e ai posteri un disegno ordinato e unitario del suo immenso corpus. Da un lato, ne aveva tracciato nell'Ordine dei miei libri il piano di una lettura sistematica, destinata anche a un possibile programma di didattica della medicina; dall'altro, nei Miei libri, aveva ricostruito la genesi del corpus, dal punto di vista della cronologia dei suoi scritti, dei loro destinatari e delle occasioni che ne avevano sollecitato la composizione.

All'estrema soglia della sua esistenza, Galeno sentì tuttavia il bisogno di un ultimo tentativo di sistemazione del suo lascito intellettuale: questa volta nella forma di una sorta di autocertificazione dottrinale, singolarmente esente da narrazioni autobiografiche, e attenta alle teorie più che alle opere in cui erano espresse. Nasceva così il singolare scritto Le mie opinioni, probabilmente l'ultimo di Galeno.

Le mie opinioni si apre con una presa di posizione di notevole vigore teorico. Non si tratta tanto di stabilire, con l'aiuto dell'autore, l'interpretazione autentica delle dottrine di Galeno. Egli assegna piuttosto all'insieme delle sue opinioni teoriche diversi livelli, o "pesi", di 
ordine epistemologico, che comportano una differenziazione nel rispettivo grado di credibilità.

$\mathrm{Ci}$ sono dunque argomenti intorno ai quali Galeno ritiene di possedere una conoscenza scientificamente sicura, una epistemoniké gnosis. Come Galeno indica a più riprese, si tratta delle questioni teoriche decidibili sulla base dell'evidenza osservativa (prevalentemente anatomica) e/o di quella razionale dei principi assiomatici, e dei procedimenti dimostrativi che ne derivano, sul modello della geometria euclidea. Ci sono poi argomenti, scientificamente indecidibili, sui quali Galeno ritiene non si possa andar oltre il livello del pithanòn, del "plausibile" o "credibile" ma non dimostrabile. E' interessante notare che questo ambito del pithanòn viene in generale riferito da Galeno a tesi platoniche di filosofia della natura, come quelle sostenute nel Timeo, che egli si sente di accettare solo dubitativamente pur senza volerle del tutto rifiutare. In qualche caso, tuttavia, può ben risultare che ciò che viene accolto come pithanòn finisca per risultare non vero, come Galeno dice a proposito della illustre tesi aristotelica della priorità del cuore nello sviluppo embrionale, che va invece rifiutata a vantaggio del fegato (cap. 11 p. 182).

Su questi argomenti è dunque possibile nutrire convinzioni scientifiche oppure credenze plausibili. C'è poi un terzo ambito di argomenti sui quali Galeno dichiara con fermezza di "non sapere nulla", perché non suscettibili né di dimostrazione scientifica né di credenze fra le quali si possa istituire una gradazione di credibilità (cap. 1).

Questa scansione epistemologica tende a sovrapporsi a un'altra distinzione: quella fra conoscenze utili alla medicina e alla filosofia morale e politica, cui spetta il livello epistemologicamente più forte, e conoscenze rilevanti solo per la "filosofia teoretica" (cfr. cap. 8 p. 180), sia nei suoi aspetti che noi diremmo 'metafisici' sia in quelli naturalistici. Le seconde comportano la formulazione di tesi scientificamente indecidibili e difficilmente argomentabili anche ai livelli della credibilità. ${ }^{1}$ Di quest'ultimo

1 La dichiarazione di Galeno in PHP IX 7.9-10 appare paradossale e persino provocatoria per un lettore delle Leggi di Platone: è impossibile decidere se il mondo sia ingenerato o generato, e, «se è generato, se ne sia stato artefice (demiourgòs) un dio oppure non vi sia nessun dio ma una causa irrazionale e priva di techne ha fabbricato il mondo, seguendo il caso (tyche) così bello come se un dio sapientissimo e potentissimo avesse presieduto alla sua formazione. Queste ricerche a nulla servono in rapporto alla buona amministrazione della propria casa o all'adeguata cura degli affari della città, o ad avere relazioni giuste e amichevoli con i parenti, i concittadini e gli stranieri». 
ambito fanno parte le questioni cosmologiche e teologiche (cap. 2 p. 173), i problemi relativi alla sostanza e all'immortalità dell'anima (capp. 7 p. 179,15 p. 189), e le grandi questioni della filosofia della natura (cap. 8 p. 180).

Sullo sfondo di queste considerazioni, vorrei ora discutere alcune delle maggiori questioni teoriche discusse nel trattato, come la teologia o la psicologia.

\section{La teologia}

La rassegna delle questioni intorno alle quali Galeno professa il proprio agnosticismo, perché scientificamente indecidibili e neppure suscettibili di ipotesi almeno persuasive, inizia con i classici problemi cosmologici: il mondo è generato o ingenerato? Ed esiste qualcosa al di fuori di esso? Dalla cosmologia Galeno passa immediatamente alla questione teologica. Egli dichiara la sua incapacità di pronunciarsi intorno alla natura, alla sostanza, al carattere incorporeo o corporeo, alla localizzazione dell'artefice del cosmo; non segue però fino in fondo il classico agnosticismo di Protagora (cap. 2 pp. 172-3), perché, a differenza del sofista, egli non dubita almeno dell'esistenza degli dèi. Ai suoi occhi, ne è prova sufficiente la loro opera provvidenziale, che viene esemplificata tanto da interventi per così dire su larga scala (la costituzione degli esseri viventi) quanto su piccola scala (presagi, sogni, intervento guaritore di Asclepio, salvataggio in mare ad opera dei Dioscuri).

La conclusione è lineare: non è di alcun danno l'ignoranza della sostanza 'metafisica' degli dèi; ciò che è utile agli uomini è dedicare loro il culto prescritto dai costumi tradizionali, come Socrate consigliava di fare con l'Apollo Pizio.

Non è difficile individuare i tratti dell'atteggiamento galenico nei riguardi della divinità. Si tratta della composizione fra due aspetti che a noi può apparire problematica, ma che tale non era affatto per Galeno, né, a dire il vero, per la gran parte degli intellettuali greci a partire da Aristotele.

Da un lato, c'è la convinzione "scientifica" dell'esistenza di un finalismo provvidenzialistico immanente alla natura, di ispirazione ancora più stoica che aristotelica perché non tollera alcuna eccezione e accentua la personificazione "divina" della natura stessa.

In numerosi passi, in Galeno il soggetto dell'ordinamento (kataskeuesis) provvidenziale è costituito dalla natura, physis: provvidenza 
divina e natura sono dunque praticamente sinonimi, appunto alla maniera stoica.

L'altro versante dell'atteggiamento di Galeno sulla questione degli dèi è quello, perfettamente socratico, dell'ossequio al culto tradizionale delle divinità cittadine, cui viene attribuita una presenza costante nella vita quotidiana, che si manifesta attraverso sogni, presagi e altri interventi benevoli (del resto, nulla di sorprendente per un personaggio come Galeno, che aveva iniziato la sua carriera all'interno del santuario di una divinità guaritrice come l'Asclepio di Pergamo).

\section{L'anima}

La questione dell'anima viene discussa a più riprese nelle Mie opinioni, ed è probabilmente quella cui Galeno dedica il maggiore impegno teorico. I temi della psicologia evocano l'intero spettro dei livelli epistemologici indicati da Galeno: la certezza scientifica, l'indecidibilità radicale, la plausibilità.

Al primo livello si colloca l'insieme delle teorie che Galeno aveva già ampiamente argomentato nel grande trattato Sulle teorie di Ippocrate e Platone. Si intende dunque per anima in primo luogo il centro delle funzioni psichiche superiori (movimento volontario, percezione, ragionamento, memoria). Seguendo l'analisi "scientifica" svolta da Platone nel libro IV della Repubblica, Galeno dà inoltre come certa la tripartizione dell'anima (cioè la sua scomposizione in una parte razionale, una emotiva e aggressiva, una terza legata ai piaceri e ai desideri corporei) e riprende dal Timeo la localizzazione delle tre parti rispettivamente nel cervello, nel cuore e nel fegato (cap. 3 p. 173 , cap. 8 p. 180). Fin qui dunque le certezze scientifiche.

Il campo sul quale Galeno professa invece la sua ignoranza, perché scientificamente incontrollabile e dimostrativamente indecidibile, è quello della "psicologia metafisica", della quale del resto viene ribadita la perfetta inutilità tanto per la medicina quanto per la filosofia morale (cap. 7 p. 179, cap. 8 p. 180). Esso comprende le questioni della natura mortale o immortale, corporea o incorporea dell'anima, sulle quali si sono affrontati platonici, aristotelici, stoici (cap. 3 p. 173). E' appena il caso di rilevare come da un punto di vista platonico la questione dell'immortalità dell'anima sia tutt'altro che irrilevante dal punto di vista morale, come mostrano i miti escatologici del Gorgia, del Fedone e del libro X della Repubblica. Il punto di vista di Galeno è qui senza dubbio 
quello di un'etica interamente "terrena" come quella di Aristotele.

Contro i sostenitori (platonici) dell'anima come sostanza autonoma e separata dal corpo, Galeno propende a credere che la "sostanza del corpo possieda la facoltà di svolgere le funzioni cui è predisposto”, incluse quelle psichiche, cioè che questa facoltà appartenga alla "sostanza attiva" (energouse ousia) del corpo stesso.

Nel capitolo 7 (p. 178), in quella che può venire considerata la sua più netta presa di posizione in senso materialistico sulla questione dell'anima, Galeno scrive: «Ritengo infatti che la generazione del corpo derivi dalla mescolanza (krasis) dei quattro elementi [aria, acqua, terra, fuoco], e se davvero l'anima è formata insieme con il corpo, essa deriva la sua generazione dalla stessa mescolanza dei quattro elementi, non potendovi essere per l'anima una generazione diversa da quella che vi è per il corpo sensibile. Credo infatti che non vi sia una sostanza (ousia) dell'anima esistente in se stessa, ma che essa sia una sorta di forma (eidos) del corpo» (cap. 7 p. 178).

Qui il rinvio implicito è all'autorità aristotelica anziché a quella platonica; ma Galeno presuppone la trasformazione della teoria dell'anima-forma in quella dell'anima come formula della mescolanza (krasis) degli elementi materiali dei corpi e dei loro organi che egli aveva già operata nello scritto Quod animi mores, Le facoltà dell'anima dell'anima dipendono dalla costituzione dei corpi (cap. 4, K. IV 782). Ora l'anima, mortale o immortale che sia, deve comunque insediarsi in un corpo per poter svolgere le sue funzioni. Allora, aggiunge Galeno, «questo mi sembra certo (saphòs): che, in quanto si insedia nei corpi, l'anima è asservita (douleuei) alla loro natura, che risulta, come dicevo, dalla peculiare mescolanza dei quattro elementi», per poi commentare in tono sarcastico: «penso che nessuno vedrà la propria competenza medica soffrire alcun danno a causa della sua ignoranza circa le cosiddette "empsicosi" e "metempsicosi"» (cap. 15 p. 188: Galeno usa qui una terminologia tecnica molto up-to-date nel linguaggio neopitagorico e neoplatonico). L'anima sta al corpo come la vista sta all'occhio: se l'occhio è in buone condizioni abbiamo una buona vista, se è leso la perdiamo, fino alla cecità (cioè alla morte).

Un ruolo importante, si è detto, tocca alla discussione della terza parte dell'anima. La sua concezione come "vegetativa" o "nutritiva" la rende del tutto estranea, come aveva già sostenuto Aristotele (Etica nicomachea I 13 1102b12-13), ai processi psichici intellettuali ed emotivi, contrariamente alla sua funzione "desiderante" in Platone. L'importanza 
della questione della terza parte dell'anima consiste appunto in questo: essa è la sede di quelle "facoltà naturali" cui Galeno aveva dedicato nell'omonimo trattato, una famosa (o famigerata) trattazione teorica. Secondo questa teoria, i principali organi somatici dispongono di un'autonoma e "spontanea" capacità di attrarre e metabolizzare le sostanze nutritive in ingresso nell'organismo, e di espellerne i residui inutili. Galeno era perfettamente consapevole della debolezza epistemologica della dottrina delle facoltà naturali: spiegare l'assimilazione di un cibo operata da un organo asserendo che questo possiede una facoltà assimilativa, significa aggirare il fatto che non se ne può enunciare scientificamente la causa (NF I 4, K. II 9-10; cfr. anche in PP cap. 14 p. 187). Questa ammirevole onestà intellettuale non induceva però Galeno a rinunciare alla centralità della teoria delle facoltà naturali. Grazie ad essa, egli poteva configurare una posizione filosofica estremamente significativa, che potremmo definire materialismo dinamico. Contro il meccanicismo atomistico da una lato, e il dualismo platonico dall'altro, e seguendo piuttosto un'ispirazione aristotelico-stoica, egli poteva cioè concepire la sostanza materiale degli esseri viventi come dotata di una sua intrinseca attività finalizzata (appunto, ousia energouse), che non richiedeva il ricorso ad entità extracorporee come l'anima-sostanza e non abbandonava d'altra parte la natura al caso e alla necessità.

\section{UNA CATASTROFE CULTURALE}

Solo un cenno, per finire, al peri alypias (L'imperturbabilità). Si tratta di un piccolo scritto in forma epistolare in cui Galeno spiega a un amico come egli non sia stato afflitto dalle perdite di beni e di libri subite nell'incendio del 192. La parte morale del trattato non presenta grandi motivi di interesse. Galeno motiva la sua serenità con uno stock di argomenti consueti ai moralisti: irrilevanza dei beni materiali, esercizio (di origine stoica) del proendemein, cioè l'assuefazione a possibili sventure rappresentate in anticipo nell'immaginazione, resistenza (se pure non paragonabile all'impossibile apatheia del saggio stoico) al dolore fisico e mentale.

L'aspetto forse più interessante dell'epistola è probabilmente costituito dal resoconto dei danni subiti personalmente da Galeno, e dall'intero patrimonio librario romano, nel corso del terribile incendio divampato nel febbraio del 192: da questo punto di vista lo scritto sul- 
l'imperturbabilità rappresenta uno straordinario documento sull'organizzazione culturale ed editoriale di Roma, e apre una finestra inedita sulle ingenti proprietà di un ricco medico romano del II secolo d.C.

L'incendio si sviluppò a partire dal tempio della Pace $(\$ 18)$, che fungeva anche da biblioteca, museo e luogo di conferenze. In seguito, esso raggiunse i magazzini siti lungo la Via Sacra, ritenuti protetti dai rischi sia del fuoco sia dei furti: qui Galeno era uso conservare i suoi beni, come in una cassaforte, e per colmo di sventura vi aveva recentemente depositato nuovi e preziosi oggetti (libri, strumenti, argenteria) che intendeva mettere al sicuro essendo in procinto di partire per la sua residenza in Campania $(\$ 10)$. Di lì il fuoco si estese alle biblioteche del Palatino ( $\$ 12 b$ ) e della Domus Tiberiana (\$18). Galeno ci offre un’importante testimonianza sulle irreparabili perdite causate dall'incendio di queste biblioteche. Vi si trovavano opere importanti di Aristotele (forse addirittura un perduto De plantis), di Teofrasto, "la maggior parte" degli scritti di Crisippo e dei medici antichi, fra i quali senza dubbio i maestri ellenistici ( $\$ 15-17)$.

All'interno di questa catastrofe culturale complessiva si inserisce poi la sventura personale di Galeno. Occorre dire che ciò che colpisce in primo luogo il lettore è la sua ingente ricchezza, che senza dubbio proveniva, in proporzioni impossibili da stabilire, sia dalle rendite delle proprietà in Asia Minore sia dall'attività professionale a Roma. Galeno possedeva una casa a Roma e una in Campania (oltre che probabilmente una terza a Pergamo), servi abbastanza numerosi da non far rimpiangere la perdita dei molti che erano stati vittime dell'epidemia di peste $(\$ 1)$, e mezzi sufficienti per disporre la costosissima riproduzione in due copie del suo enorme corpus di scritti, ${ }^{2}$ una delle quali destinata alla casa in Campania, l'altra a venir donata a una biblioteca di Pergamo (\$21).

Non può dunque sorprendere l'impressionante quantità di beni la cui perdita viene inventariata nella lettera all'amico turbato. Essi possono venire ripartiti in tre gruppi.

Beni materiali. Nei magazzini della Via Sacra Galeno aveva in primo luogo depositato i classici beni da cassaforte: ori, argenti, certifi-

2 V. Nutton stima che i soli scritti di Galeno devono aver occupato almeno sei o settecento rotoli: cfr. V. Nutton, Galen's library, in Galen and the World ok Knowledge, a cura di Ch.Gill-T. Whitmarsh-J. Wilkins, Cambridge Univ. Press, Cambridge 2009, pp. 19-34. 
cati di credito $(\$ 4)$. Ma c'erano anche strumenti di impiego medico, alcuni dei quali progettati e fatti costruire dallo stesso Galeno ( $\$ 5)$ : si trattava presumibilmente di strumenti chirurgici e anatomici, tra cui alcuni destinati a fini di ricerca. ${ }^{3}$ In questo gruppo, la perdita più dolorosa era quella di una gran quantità di farmaci rari (\$6). Sappiamo che per raccoglierne gli ingredienti, minerali (bitume, cadmio, rame) e vegetali, in quantità tale che gli bastasse per tutta la vita, Galeno aveva viaggiato in Siria, Palestina, Cipro, Licia e a Lemno. In particolare, nella Via Sacra era custodito un'enorme deposito di cinnamomo (cassia) e del celebre farmaco detto "teriaca", composto di non meno di 70 ingredienti, ${ }^{4}$ il cui valore era senza dubbio assai ingente.

I libri. Per noi, e senza dubbio anche per lo stesso Galeno, è molto più penosa la perdita di libri. Il deposito della Via Sacra ne conteneva molti, soprattutto quelli inviativi di recente in vista della partenza per la Campania. La biblioteca perduta di Galeno può venire suddivisa in tre sezioni principali.

(A) La prima sezione comprendeva le copie, fatte eseguire o eseguite personalmente da Galeno, di libri rari (come alcuni di Teofrasto e forse di Aristotele) o in edizione pregiata (come il Platone di Panezio) $(\$ 13,17)$. (B) La seconda sezione era composta dai testi - tra cui quelli

3 Sugli strumenti anatomici di Galeno cfr. I. Garofalo, Introduzione a Procedimenti anatomici, BUR, Milano 1991, pp. 55-57. In questa opera Galeno racconta di un semplice strumento (un tubetto di bronzo) adibito a mostrare la presenza di sangue nelle arterie, contro i seguaci di Erasistrato; uno dei suoi rivali avrebbe progettato uno strumento molto più complesso (una scure a quattro lame, peraltro mai realizzata) per provare la tesi contraria: $A A$ VII 16, K. II 643-46. Alcuni degli strumenti chirurgici descritti da Galeno sono ora riconoscibili fra quelli reperiti nella "Domus del chirurgo" (III sec. d.C) recentemente messa in luce a Rimini: cfr. R. Jackson, Lo strumentario chirurgico della domus riminese, in Ars medica. I ferri del mestiere: la domus del chirurgo e la chirurgia nell'antica Roma, a cura di S. De Carolis, Guaraldi, Rimini 2009, pp. 73-91. Nello stesso sito sono stati reperiti grandi contenitori per la preparazione e la conservazione dei farmaci, probabilmente simili a quelli depositati da Galeno nei magazzini della Via Sacra.

4 Il principale dei quali era la carne di vipera, ma c'era anche una significativa presenza di oppio. Destinata in origine a curare i morsi degli animali velenosi, l'azione benefica della teriaca (forse soprattutto dovuta agli oppiacei) si era estesa a una quarantina di malattie. Sull'autenticità dello scritto galenico Theriaca ad Pisonem cfr. V. Nutton, Galen on theriac: problems of authenticity, e sulla composizione del farmaco M. Stein, La thériaque chez Galien: sa préparation et son usage thérapeutique, entrambi in Galen on Pharmacology, a cura di A. Debru, Brill, Leiden 1997, pp. 85-102; 199-209. 
dei peripatetici, di Crisippo, dei medici antichi - sui quali Galeno aveva condotto un lavoro di accurata revisione editoriale $(\$ 14-15)$. (C) La terza sezione comprendeva le opere di Galeno di cui era stata avviata la trascrizione in due copie. In alcuni casi, come il grande lessico tratto dalla commedia antica, la perdita risultò irrimediabile perché andarono perduti anche gli originali $(\$ 23 \mathrm{~b})$, al pari di quelli delle molte sinossi che Galeno aveva compilato per uso personale (\$30); in altri Galeno fu costretto a riscrivere il suo testo; altri scritti ancora risultarono tuttavia recuperabili perché ne erano sopravvissute copie già in circolazione.

Le ricette. Galeno attribuisce un particolare rilievo alla perdita di preziose raccolte di ricette, trascritte in tre grandi volumi pergamenacei, che egli aveva ereditato da amici e che usava anche come mezzo di scambio per acquisirne di nuove (\$31-36). Si trattava di farmaci rari, mirabili (thaumasia) e celebrati (eudokimounta), la cui conoscenza contribuiva certamente al prestigio di Galeno presso i suoi ricchi pazienti desiderosi di giovarsi di medicine tanto costose quanto strabilianti.

L'enormità di queste perdite, serenamente sopportate, doveva costituire secondo Galeno la miglior prova che si potesse dare all'amico della sua forza d'animo. E questa a sua volta è documento tanto della buona "natura" che egli aveva ereditato dalla famiglia, tanto dell'efficacia di quegli "esercizi, discorsi e dottrine" con cui nel tempo aveva coltivato la sua disposizione naturale, preparandola a resistere agli eventi della vita senza subirne un'eccessiva afflizione. Fra questi esercizi, è degna di particolare menzione la preparazione di Galeno ad affrontare la condanna all'esilio o alla morte così comune nell'epoca sciagurata dell'impero di Commodo (\$54-55): si tratta della più antica e violenta critica a noi nota a questo imperatore (che da giovane era stato paziente di Galeno, cui l'aveva affidato il padre Marco Aurelio), in un'opera scritta pochi mesi dopo la sua morte, all'inizio del 193, e questo costituisce un altro motivo dell'interesse documentario presentato dal piccolo scritto sulla Imperturbabilità. 